\title{
Imidazo[2,1-b]benzothiazol Derivatives as Potential Allosteric Inhibitors of the Glucocorticoid Receptor
}

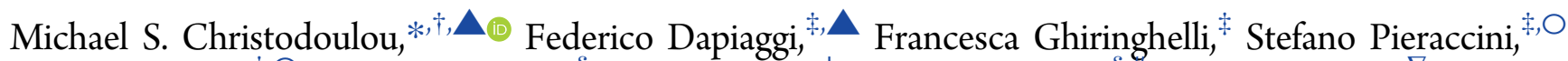
Maurizio Sironi, ${ }^{\ddagger} \bigcirc$ Marianna Lucafò, ${ }^{\S}$ Debora Curci, ${ }^{\perp}$ Giuliana Decorti, ${ }^{\S, \#}$ Gabriele Stocco, ${ }^{\nabla}$ Chandra Sekhar Chirumamilla, Wim Vanden Berghe, ${ }^{\bullet}$ Patrick Balaguer, $^{\text {II }}$ Benoît Y. Michel, $\square$ Alain Burger, ${ }^{\square}$ Egle M. Beccalli, ${ }^{\dagger}$ Daniele Passarella, ${ }^{\ddagger}{ }^{\circ}$ and Nadine Martinet ${ }^{*}, \square$

${ }^{\dagger}$ DISFARM, Sezione di Chimica Generale e Organica “A. Marchesini”, Università degli Studi di Milano, Via Venezian 21, 20133 Milano, Italy

${ }^{\ddagger}$ Department of Chemistry, Università degli Studi di Milano, Via Golgi 19, 20133 Milano Italy

${ }^{\S}$ Department of Medicine, Surgery and Health Sciences, University of Trieste, 34127 Trieste, Italy

${ }^{\perp} \mathrm{PhD}$ School in Reproduction and Developmental Sciences, University of Trieste, 34127 Trieste, Italy

\#Institute for Maternal and Child Health - IRCCS "Burlo Garofolo", 34127 Trieste, Italy

$\nabla^{D}$ Department of Life Sciences, University of Trieste, 34127 Trieste, Italy

OIstituto di Scienze e Tecnologie Molecolari (INSTM), CNR, and INSTM, UdR Milano, Via Golgi 19, 20133 Milano, Italy

Laboratory of Protein Chemistry, Proteomics and Epigenetic Signalling, Department of Biomedical Sciences, University of Antwerp (UA), 2000 Antwerpen, Belgium

IIRCM, INSERM U1194, Université Montpellier, ICM, 208 rue des Apothicaires, F-34298 Montpellier, France

$\square$ Université Côte d'Azur, CNRS, Institut de Chimie de Nice, UMR 7272, Parc Valrose, 06108 Nice Cedex 2, France

\section{Supporting Information}

ABSTRACT: Glucocorticoid receptor (GCR) transactivation reporter gene assays were used as an initial high-throughput screening on a diversified library of 1200 compounds for their evaluation as GCR antagonists. A class of imidazo $[2,1-b]$ benzothiazole and imidazo $[2,1-b]$ benzoimidazole derivatives were identified for their ability to modulate GCR transactivation and antiinflammatory transrepression effects utilizing GCR and NF- $\kappa \mathrm{B}$ specific reporter gene assays. Modeling studies on the crystallographic structure of the GCR ligand binding domain provided three new analogues bearing the tetrahydroimidazo[2,1-b] benzothiazole scaffold able to antagonize the GCR in the presence of dexamethasone (DEX) and also defined their putative binding into the GCR structure. Both mRNA level measures of GCR itself and its target gene GILZ, on cells treated with the new analogues, showed a GCR transactivation inhibition, thus suggesting a potential allosteric inhibition of the GCR.

KEYWORDS: Imidazo[2,1-b]benzothiazole, imidazo[2,1-b]benzoimidazole, GCR allosteric inhibition, anti-inflammatory GCR like activity, reduced GILZ expression

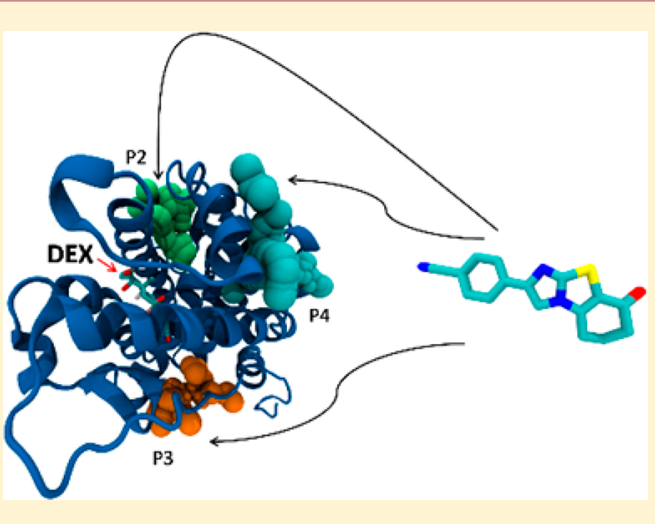

$\mathrm{C}$ ortisol is synthesized in the adrenal glands but is also regenerated mainly in the liver from inactive cortisone by $11 \beta$-hydroxysteroid dehydrogenase 1 . Natural and synthetic glucorticoids are the ligands of the glucocorticoid receptor (GCR), which belongs to the nuclear hormone receptor superfamily of ligand-activated transcription factors. GCR has a dual mode of action: "transactivation" as a ligand-activated transcription factor that binds to glucocorticoid response elements located in the nuclear and mitochondrial DNA and as a modulator (often trans-repressor) of other transcription factors such as NF-kB. ${ }^{1-3}$ The final resulting physiological action is the regulation/maintenance of basal and stress-related homeostasis. ${ }^{4}$

Glucocorticoids are the most prescribed drugs for antiinflammatory purposes, but their continued use is restricted by serious side effects: hypertension and major metabolic side effects such as glucose intolerance, muscle wasting, skin thinning, and osteoporosis. Therefore, in order to avoid such side effects, GCR signaling pathways have been the focus of

Received: December 19, 2017

Accepted: February 26, 2018

Published: February 26, 2018 
intensive research to find modulators active either on transactivation or transrepression. ${ }^{5-7}$

Selective GCR antagonists have been the interest of active chemistry in the last two decades. Their potential therapeutic applications are very broad, including Cushing's syndrome, psychotic depression, diabetes, obesity, Alzheimer's disease, neuropathic pain, drug abuse, and glaucoma. ${ }^{8}$ Depending on the chemical structure, they can be classified as (1) steroidal, such as the nonselective GCR antagonist RU-486 (mifepristone), which was used for the treatment of Cushing's syndrome ${ }^{9}$ and the GCR-selective steroid RU-43044 ${ }^{10}$ and (2) nonsteroidal GCR antagonists including octahydrophenanthrenes, spirocyclic dihydropyridines, triphenylmethanes and diaryl ethers, chromenes, dibenzyl anilines, dihydroisoquinolines, pyrimidinediones, azadecalins, aryl pyrazolo azadecalins, quinolin-3-one, and polyhydroxylated polychlorinated biphenyls. ${ }^{8,11-14}$

Herein, by screening a library of 1200 compounds using GCR competition binding assays, a new moiety bearing the imidazo[2,1-b]benzothiazole and imidazo[2,1-b]benzoimidazole skeleton was identified as a new scaffold for the inhibition of the GCR activity (Figure 1). Modeling studies

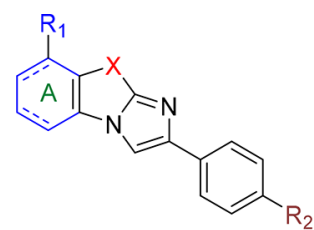

Hit compounds

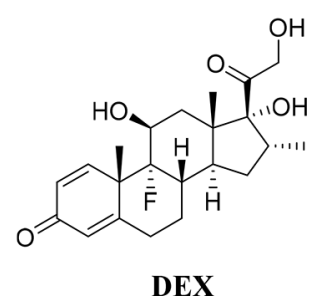

DEX
Figure 1. General structure of the hit compounds and of DEX.

of the most active compound on the crystallographic structure of the GCR ligand binding domain provided three novel structures, which were synthesized and further evaluated for their antagonist action mechanism against GCR in the presence of $\mathrm{DEX}^{19}$ (Figure 1). Allosteric binding and inhibition of inflammatory activity were the two main suggested features for our compounds.

First, GCR luciferase reporter gene assay was performed following single compound treatment $(0.1 \mu \mathrm{M})$ to compare potential agonistic compound effects relative to the reference agonistic compound DEX $(1 \mu \mathrm{M})$. However, none of the tested compounds induced GCR agonistic effects (Figure S1). Next, to evaluate antagonistic effects, we performed a GRE luc reporter gene assay following single compound combination treatments with DEX $(1 \mu \mathrm{M})$. As it can be observed from Table 1 , DEX induced GCR transactivation can be reversed by compounds 1-9, with compound 9 being the most potent, reducing DEX induced GCR transactivation by $82 \%$ (100\% is DEX effect). In contrast, a compound with a similar structure, 10, was found to be completely ineffective (Table 1, Figure S2). The increased effect of the hydrochloric salts $\mathbf{1}$ and $\mathbf{6}$, in comparison to the parent compounds 10 and 2 , can be explained by the higher water solubility.

Complementary to the GCR reporter screening, the most active compounds identified in the GCR reporter assay were also tested for their possible anti-inflammatory GCR transrepression effects in a complementary NF-kB reporter assay. Compounds were evaluated for their potency to suppress TNF induced NF-kB reporter activity upon $4 \mathrm{~h}$ combination
Table 1. GCR Antagonistic Effects in Comparison to DEX Effects Taken as $\mathbf{1 0 0 \%}$

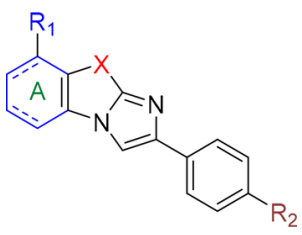

\begin{tabular}{|c|c|c|c|c|c|c|}
\hline compd & A & $\mathrm{X}$ & $\mathrm{R}_{1}$ & $\mathrm{R}_{2}$ & $\mathrm{GCR}^{a}$ & $\begin{array}{c}\mathrm{HeLa}^{b} \\
\mathrm{EC}_{50}(\mu \mathrm{M})\end{array}$ \\
\hline 1 & & $S$ & $\mathrm{H}$ & $\mathrm{CH}_{2} \mathrm{NH}_{2} \cdot \mathrm{HCl}$ & $38 \pm 5$ & \\
\hline 2 & & S & $\mathrm{H}$ & $\mathrm{CH}_{2} \mathrm{NH}_{2}$ & $35 \pm 11$ & \\
\hline 3 & & S & $\mathrm{H}$ & $\mathrm{CH}_{2} \mathrm{OH}$ & $34 \pm 14$ & \\
\hline 4 & & $S$ & $\mathrm{H}$ & $\mathrm{CH}_{3}$ & $30 \pm 1$ & \\
\hline 5 & & NEt & $\mathrm{H}$ & $\mathrm{CN}$ & $25 \pm 1$ & \\
\hline 6 & & $S$ & $\mathrm{H}$ & $\mathrm{CH}_{2} \mathrm{NH}_{2} \cdot \mathrm{HCl}$ & $23 \pm 2$ & \\
\hline 7 & & S & $\mathrm{H}$ & $\mathrm{NH}_{2} \cdot \mathrm{HCl}$ & $23 \pm 3$ & \\
\hline 8 & & $S$ & $\mathrm{H}$ & $\mathrm{Br}$ & $20 \pm 1$ & \\
\hline 9 & & $S$ & $\mathrm{H}$ & $\mathrm{CN}$ & $18 \pm 3$ & $1.7 \pm 1.0$ \\
\hline 10 & & S & $\mathrm{H}$ & $\mathrm{CH}_{2} \mathrm{NH}_{2}$ & $138 \pm 3$ & $3.6 \pm 1.3$ \\
\hline 11 & & $S$ & $\mathrm{H}$ & $\mathrm{COOH}$ & & $170 \pm 1.08$ \\
\hline 12 & & $S$ & $\mathrm{H}$ & $\mathrm{COOCH}_{3}$ & & $7.7 \pm 1.1$ \\
\hline 13 & & $S$ & 足 & $\mathrm{CN}$ & & $16 \pm 1.1$ \\
\hline
\end{tabular}

${ }^{a}$ Modulation in $\% .{ }^{b}$ The data are reported as means \pm SE of three independent experiments performed in triplicate.

treatment following $2 \mathrm{~h}$ pretreatment with the single compound. Interestingly, various degrees of NF-kB inhibition were observed, with compound 9 being the most bioactive, reducing TNF induced NF-kB activation by approximately $76 \%$ (Figure 2).

It is known that molecular docking studies on biological systems can predict the structures of intermolecular complexes formed by ligands and their receptors. ${ }^{16}$ In a number of different systems, molecular docking has been employed to rationalize experimental results for a great variety of targets, such as proteins, ${ }^{17-19}$ enzymes, ${ }^{20-23}$ and DNA. ${ }^{24-26}$ The technique can predict intermolecular receptor-ligand structures not easily accessible through experiments. Moreover, molecular docking can provide atomic level information about the interactions occurring within the binding site and in the putative ligand-binding "pockets" found in a protein.

Here, the crystallographic structure of the GCR ligand binding domain revealed 14 such different pockets, whose volume, depth, and polarity are shown in Supporting Information Table S1. A molecular docking calculation was carried out for the most antagonistic compound, 9, in every 


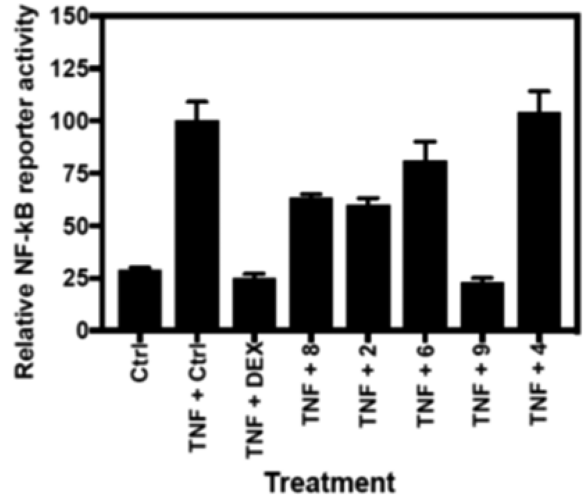

Figure 2. Suppression of TNF induced NF-kB reporter activity in the presence of tested compounds.

pocket of the GCR (Table S2). The most interesting pockets, from the geometric, energetic, and chemical points of view, were found to be pockets 2,3 , and 4 . In these pockets, indeed, we noticed a high density of hydrogen bond donor residues (Table S3). This common feature of the three pockets led us to the design of three new compounds: 11, 12, and $\mathbf{1 3}$ (Table 1). These molecules share the same scaffold with compound 9 but have different $R_{1}$ and $R_{2}$ substituents. In particular, we added hydrogen bond acceptor functional groups, such as carboxyl, ester, and carbonyl group, in order to improve the binding in pockets 2, 3, and 4, exploiting the high density of hydrogen bond donor residues. These new compounds were docked in all the 14 pockets identified by the former analysis in order to compare their binding poses and energy (Table S2). Interestingly, compounds 11, 12, and 13 always displayed a lower binding energy compared to 9 , in particular in pockets 2 , 3 , and 4 .

The binding site of DEX (pocket 0) showed the largest volume and the lowest binding energy with respect to the other pockets confirming that, in the absence of DEX, the studied compounds would bind in this pocket but with a lower affinity comparing to DEX. The docked conformation of the modeled compounds displayed the lowest energy values in pockets 2,3 , and 4 highlighted in Figure 3, where the compounds are shown as being able to bind even in the presence of DEX. We carried out an analysis of chemical interactions in the selected pockets. All the docked structures in pocket 2 adopt the same binding mode. The carboxyl and the ester of compounds 11 and 12 are

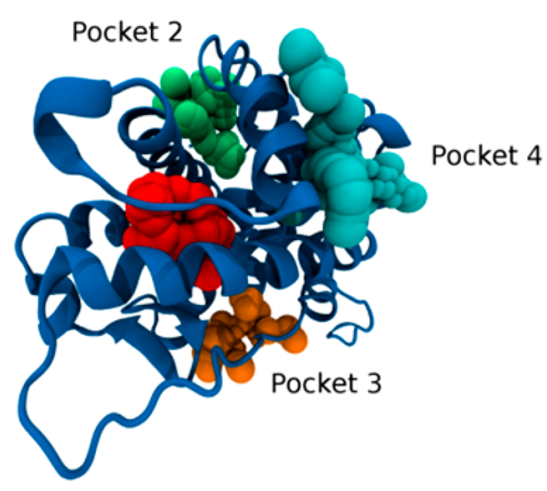

Figure 3. Three putative binding sites in the ligand binding domain of the glucocorticoid receptor for compounds 11, 12, and 13: in green, pocket 2; in cyan, pocket 4 ; in orange, pocket 3 ; in red, the binding site of DEX. oriented toward His 726, Tyr 764, and Ser 674, with the two oxygens forming hydrogen bonds (HBs) with the aforementioned residues (Figure S3). The nitrile substituents of compounds 9 and $\mathbf{1 3}$ form a HB with His 726 (Figure S4). The docking poses of the same compounds within pocket 3 are, also, very similar. We observed a salt bridge between the carboxyl group of compound 11 and Arg 614, and a HB between the acceptor nitrogen of all scaffolds and Tyr 663 (Figure S5). Within pocket 4, compound 9 exhibited a different docking pose compared to 11,12 , and 13. In particular, there is a salt bridge between the carboxyl group of 11 and Arg 585, and a HB between both the carbonyl groups of 11 and 12 and Gln 592. The carbonyl group of compound 13 makes a HB with Met 752 (Figure S6). The nitrile group of 9 is oriented toward the same direction, but the docking pose of the rigid scaffold differs from 11, 12, and 13 (Figure S7). These three compounds were designed in such a way as to interact with $\mathrm{HB}$ donor residues in the selected pockets (Table S3), providing additional interactions compared to compound 9. Finally, all the modified compounds display a lower binding energy in the selected pockets with respect to compound 9.

In the next step, the proposed compounds 11-13 were tested for their GCR antagonist activity. The results presented in Supporting Information Figure S8 show that, unlike RU486, which competitively inhibits GCR activity, the compounds 1113 are not classic competitive inhibitors of the GCR activity since they do not compete with DEX.

Since our compounds showed non-competitive antagonism against DEX, we evaluated the cytotoxic effects on epithelial cells of the most active compounds 9 and 11-13; compound 10 was our negative control since it showed very low activity in terms of binding to the GCR (Table 1). The cytotoxicity of derivatives 9, 10, 11, 12, and 13 for HeLa cells was studied with the MTT test, after $72 \mathrm{~h}$ of cell exposure in complete medium. Data presented in Table 1 and Figure 4 show that all the

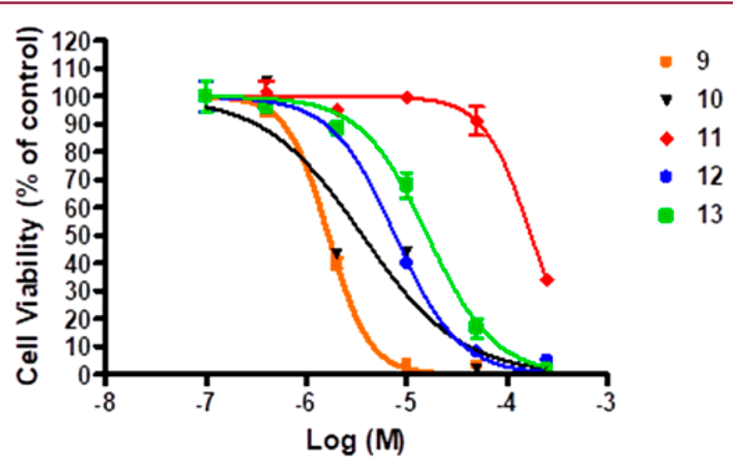

Figure 4. Effect of compounds 9, 10, 11, 12, and 13 in HeLa cells. Cells were exposed for $72 \mathrm{~h}$ to the compounds at different concentrations $(0.4,2,10,50$, and $250 \mu \mathrm{M})$, and cytotoxicity was evaluated by the MTT assay. The data are reported as means \pm SE of three independent experiments performed in triplicate.

compounds induced a cytotoxic effect with an $\mathrm{EC}_{50}(\mu \mathrm{M})$ of $1.7,3.6,170,7.7$, and 16 for compounds $9,10,11,12$, and 13, respectively. In terms of cytotoxicity for HeLa cells, these results demonstrate a pronounced difference in the behavior of the 9 and 10 derivatives and 11, 12, and 13 compounds tested. Further analysis was therefore focused on the less toxic compounds 11, 12, and 13 (Figure 4).

To investigate the mechanism of the non-competitive antagonism found, the effect of compounds 11, 12, and 13 
on GCR expression was evaluated. GCR transcripts were quantified in HeLa cells treated for $24 \mathrm{~h}$ at 0.4 and $2 \mu \mathrm{M}$ with the three compounds. Interestingly, the treatment with the compounds at $0.4 \mu \mathrm{M}$ induced a downregulation of the GCR (about $50 \%$ for compound 11), but not at $2 \mu \mathrm{M}$ (Figure 5).

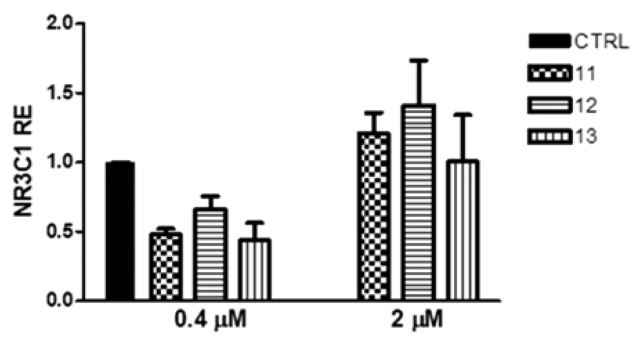

Figure 5. GCR expression in HeLa cells after treatment with compounds 11, 12, and 13 for $24 \mathrm{~h}$ at 0.4 and $2 \mu \mathrm{M}$. One-way ANOVA; $0.4 \mu \mathrm{M}, p=0.057 ; 2 \mu \mathrm{M}, p=0.92$. The data are reported as means \pm SD of three independent experiments.

The presence of this effect only at lower concentration could be related to cytotoxic effects at higher concentration. The effect on GCR expression of compounds $\mathbf{9}$ and $\mathbf{1 0}$ was also evaluated (Figure S9), highlighting that the least cytotoxic compound (9) is more similar to compounds 11-13 than compound 10 .

GILZ is one of the earliest and most GCR inducible genes by transactivation. To confirm the ability of compounds 11, 12, and 13 to modulate GCR transactivation, the expression of GILZ was measured in HeLa cells treated for $24 \mathrm{~h}$ with compounds 11, 12, and 13 at 0.4 and $2 \mu \mathrm{M}$. As shown in Figure 6 , the compounds induced a downregulation of GILZ

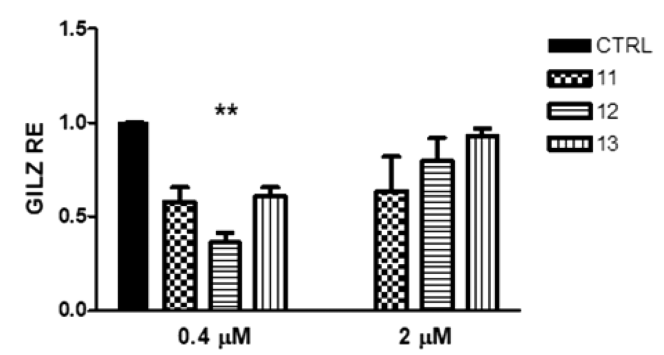

Figure 6. GILZ expression in HeLa cells after treatment with 11, 12, and 13 for $24 \mathrm{~h}$ at 0.4 and $2 \mu \mathrm{M}$. One-way ANOVA; $0.4 \mu \mathrm{M}, p=$ $0.0090 ; 2 \mu \mathrm{M}, p=0.58$; Bonferroni post-test 12 vs CTRL, $* * p$-value < 0.001 . The data are reported as means \pm SD of three independent experiments.

expression, by comparison to untreated cells (CTRL), indicating that our compounds could prevent the transactivation of the GCR. The strongest effect was observed for compound 12. GILZ downregulation was evident at lower concentration tested $(0.4 \mu \mathrm{M})$, likely because at $2 \mu \mathrm{M}$ the compounds start to induce a cytotoxic activity. To verify further the inhibition of GCR-induced transactivation, HeLa cells were also treated with DEX alone at $0.1 \mu \mathrm{M}$ and in combination with compounds 11, 12, and 13 for $24 \mathrm{~h}$ (Figure 7). As a positive control, the GCR competitive antagonist RU486 was used in combination with DEX (Figure 7). Cotreatment of cells with DEX and RU486 showed that DEX-induced GCR-mediated GILZ transcription was inhibited as expected. Similar results were obtained when cells were treated with compounds 11, 12, and 13 plus DEX, with compound 12 showing again the most significant effect and likewise demonstrated the ability of this

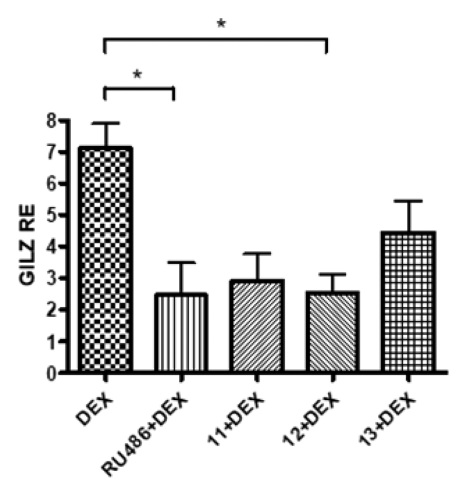

Figure 7. GILZ expression in HeLa cells after treatment with DEX alone at $0.1 \mu \mathrm{M}$, with RU486 $0.4 \mu \mathrm{M}$ in combination with DEX and with 11, 12, and 13 at $0.4 \mu \mathrm{M}$ in cotreatment with DEX for $24 \mathrm{~h}$. Oneway ANOVA, $p=0.011$; Bonferroni post-test: RU486 + DEX vs DEX, ${ }^{*} p$-value $<0.05 ; 12+\mathrm{DEX}$ vs DEX, $* p$-value $<0.05$. The data are reported as means $\pm \mathrm{SD}$ of three independent experiments.

compound to inhibit the transcriptional activity of the GCR. The effect on GILZ expression of compounds 9 and 10 was also evaluated (Figure S10), highlighting that no significant inactivation of GCR is induced by these compounds since GILZ levels are not affected.

While in the GILZ expression assay, the compounds reduced DEX activity with an effect similar to RU486 (Figure 7), a competitive inhibitor of GCR; results from GCR reporter assay (Figure S8) indicate that the antagonism of our compounds is not surmountable by increasing DEX concentration and therefore occurs through a non-competitive mechanism.

In this letter, through an in vitro screening of 1200 compounds using a GCR reporter gene assay, nine molecules were identified as potent GCR antagonists. In addition, some analogs maintained an anti-inflammatory GCR-like activity as revealed by our NF-kB reporter gene studies. Molecular docking on the most active compound revealed the presence of three pockets suitable for binding. Based on the high density of $\mathrm{HB}$ donor residues found in these pockets, three novel compounds were designed. All the modified compounds displayed a lower binding energy in the selected pockets compared to the parent compound. The biological evaluation of the synthesized novel molecules did not show a classic antagonism against GCR but rather a reverse GCR transactivation, illustrated with a reduced expression of the GILZ gene. However, in contrast to RU486, our molecules failed to displace DEX in ligand binding assays. In silico studies provided three putative binding sites (pockets) in the ligand binding domain of the GCR where the compounds can bind in the presence of DEX.

Taking into account all the experimental data, we conclude that our novel analogues hold promise as a novel class of antiinflammatory GCR modulator compounds with decreased GCR transactivation properties. Most importantly, given that they bind to the GCR but do not displace the reference ligand DEX, ${ }^{27,28}$ their allosteric binding is the most likely explanation.

These results open the door for designing improved antiinflammatory GCR modulators with reduced side effects. 


\section{ASSOCIATED CONTENT}

\section{S Supporting Information}

The Supporting Information is available free of charge on the ACS Publications website at DOI: 10.1021/acsmedchemlett.7b00527.

Preparation of compounds 11-13; in silico studies; description of in vitro evaluation GCR and NF-kB reporter gene studies; cell viability analysis; RNA isolation and quantitative real-time PCR (TaqMan); Supplementary Figures, Tables, and Schemes (PDF)

\section{AUTHOR INFORMATION}

\section{Corresponding Authors}

*E-mail: michail.christodoulou@unimi.it.

*E-mail: nadine.martinet1@free.fr.

\section{ORCID}

Michael S. Christodoulou: 0000-0002-5098-3143

Wim Vanden Berghe: 0000-0003-0161-7355

Daniele Passarella: 0000-0001-6180-9581

\section{Author Contributions}

$\boldsymbol{\Delta}_{\text {These authors contributed equally. The manuscript was }}$ written through contributions of all authors. All authors have given approval to the final version of the manuscript.

\section{Notes}

The authors declare no competing financial interest.

\section{ACKNOWLEDGMENTS}

This research has been developed under the umbrella of CM1106 COST Action "Chemical Approaches for Targeting Drug Resistance in Cancer Stem Cells". The authors express their gratitude to Ms. Ioana Stupariu for the revision of the manuscript.

\section{REFERENCES}

(1) Ratman, D.; Vanden Berghe, W.; Dejager, L.; Libert, C.; Tavernier, J.; Beck, I. M.; De Bosscher, K. How glucocorticoid receptors modulate the activity of other transcription factors: a scope beyond tethering. Mol. Cell. Endocrinol. 2013, 380, 41-54.

(2) De Bosscher, K.; Vanden Berghe, W.; Haegeman, G. Cross-talk between nuclear receptors and nuclear factor kappaB. Oncogene 2006, $25,6868-6886$.

(3) De Bosscher, K.; Vanden Berghe, W.; Haegeman, G. The Interplay between the Glucocorticoid Receptor and Nuclear Factor-kB or Activator Protein-1: Molecular Mechanisms for Gene Repression. Endocr. Rev. 2003, 24, 488-522.

(4) Vandevyver, S.; Dejager, L.; Libert, C. Comprehensive overview of the structure and regulation of the glucocorticoid receptor. Endocr. Rev. 2014, 35, 671-693.

(5) Newton, R. Anti-inflammatory glucocorticoids: changing concepts. Eur. J. Pharmacol. 2014, 724, 231-236.

(6) De Bosscher, K.; Beck, I. M.; Ratman, D.; Vanden Berghe, W.; Libert, C. Activation of the Glucocorticoid Receptor in Acute Inflammation: the SEDIGRAM Concept. Trends Pharmacol. Sci. 2016, 37, 4-16.

(7) Chirumamilla, C. S.; Palagani, A.; Kamaraj, B.; Declerck, K.; Verbeek, M. W. C.; Oksana, R.; De Bosscher, K.; Bougarne, N.; Ruttens, B.; Gevaert, K.; Houtman, R.; De Vos, W. H.; Joossens, J.; Van Der Veken, P.; Augustyns, K.; Van Ostade, X.; Bogaerts, A.; De Winter, H.; Vanden Berghe, W. Selective Glucocorticoid Receptor Properties of GSK866 Analogs with Cysteine Reactive Warheads. Front. Immunol. 2017, 8, 1324.

(8) Clark, R. D. Glucocorticoid receptor antagonists. Curr. Top. Med. Chem. 2008, 8, 813-838.
(9) Nieman, L. K.; Chrousos, G. P.; Kellner, C.; Spitz, I. M.; Nisula, B. C.; Cutler, G. B.; Merriam, G. R.; Bardin, C. W.; Loriaux, D. L. Successful Treatment of Cushing's Syndrome with the Glucocorticoid Antagonist RU 486. J. Clin. Endocrinol. Metab. 1985, 61, 536-540.

(10) Teutsch, G.; Gaillard-Moguilewsky, M.; Lemoine, G.; Nique, F.; Philibert, D. Design of ligands for the glucocorticoid and progestin receptors. Biochem. Soc. Trans. 1991, 19, 901-908.

(11) Eda, M.; Kuroda, T.; Kaneko, S.; Aoki, Y.; Yamashita, M.; Okumura, C.; Ikeda, Y.; Ohbora, T.; Sakaue, M.; Koyama, N.; Aritomo, K. Synthesis and Biological Evaluation of Cyclopentaquinoline Derivatives as Nonsteroidal Glucocorticoid Receptor Antagonists. J. Med. Chem. 2015, 58, 4918-4926.

(12) Takeuchi, S.; Shiraishi, F.; Kitamura, S.; Kuroki, H.; Jin, K.; Kojima, H. Characterization of steroid hormone receptor activities in 100 hydroxylated polychlorinated biphenyls, including congeners identified in humans. Toxicology 2011, 289, 112-121.

(13) Jin, Z.; Lin, H.; Srinivasan, S.; Nwachukwu, J. C.; Bruno, N.; Griffin, P. R.; Nettles, K. W.; Kamenecka, T. M. Synthesis of novel steroidal agonists, partial agonists, and antagonists for the glucocorticoid receptor. Bioorg. Med. Chem. Lett. 2017, 27, 347-353.

(14) Morgan, B. P.; Swick, A. G.; Hargrove, D. M.; LaFlamme, J. A.; Moynihan, M. S.; Carroll, R. S.; Martin, K. A.; Lee, E.; Decosta, D.; Bordner, J. Discovery of Potent, Nonsteroidal, and Highly Selective Glucocorticoid Receptor Antagonists. J. Med. Chem. 2002, 45, 24172424.

(15) Lucafò, M.; Stankovic, B.; Kotur, N.; Silvestre, A. D.; Martelossi, S.; Ventura, A.; Zukic, B.; Pavlovic, S.; Decorti, G. Pharmacotranscriptomic biomarkers in glucocorticoid treatment of pediatric inflammatory bowel disease. Curr. Med. Chem. 2017, DOI: 10.2174/ 0929867324666170920145337.

(16) Yuriev, E.; Agostino, M.; Ramsland, P. A. Challenges and advances in computational docking: 2009 in review. J. Mol. Recognit. 2011, 24, 149-164.

(17) Marucci, C.; Christodoulou, M. S.; Pieraccini, S.; Sironi, M.; Dapiaggi, F.; Cartelli, D.; Calogero, A. M.; Cappelletti, G.; Vilanova, C.; Gazzola, S.; Broggini, G.; Passarella, D. Synthesis of PironetinDumetorine Hybrids as Tubulin Binders. Eur. J. Org. Chem. 2016, 11, 2029-2036.

(18) Christodoulou, M. S.; Caporuscio, F.; Restelli, V.; Carlino, L.; Cannazza, G.; Costanzi, E.; Citti, C.; Lo Presti, L.; Pisani, P.; Battistutta, R.; Broggini, M.; Passarella, D.; Rastelli, G. Probing an allosteric pocket of CDK2 with small-molecules. ChemMedChem 2017, $12,33-41$.

(19) Christodoulou, M. S.; Mori, M.; Pantano, R.; Alfonsi, R.; Infante, P.; Botta, M.; Damia, G.; Ricci, F.; Sotiropoulou, P. A.; Liekens, S.; Botta, B.; Passarella, D. Click Reaction as a Tool to Combine Pharmacophores: The Case of Vismodegib. ChemPlusChem 2015, 80, 938-943.

(20) Laco, G. S.; Collins, J. R.; Luke, B. T.; Kroth, H.; Sayer, J. M.; Jerina, D. M.; Pommier, Y. Human topoisomerase I inhibition: docking camptothecin and derivatives into a structure-based active site model. Biochemistry 2002, 41, 1428-1435.

(21) Christodoulou, M. S.; Zarate, M.; Ricci, F.; Damia, G.; Pieraccini, S.; Dapiaggi, F.; Sironi, M.; Lo Presti, L.; García-Argáez, A. N.; Dalla Via, L.; Passarella, D. 4-(1, 2-diarylbut-1-en-1-yl)isobutyranilide derivatives as inhibitors of topoisomerase II. Eur. J. Med. Chem. 2016, 118, 79-89.

(22) Navakauskienè, R.; Mori, M.; Christodoulou, M. S.; Zentelytè, A.; Botta, B.; Dalla Via, L.; Ricci, F.; Damia, G.; Passarella, D.; Zilio, C.; Martinet, N. Histone demethylating agents as potential S-adenosylL-methionine-competitors. MedChem Comm 2016, 7, 1245-1255.

(23) Christodoulou, M. S.; Zunino, F.; Zuco, V.; Borrelli, S.; Comi, D.; Fontana, G.; Martinelli, M.; Lorens, J. B.; Evensen, L.; Sironi, M.; Pieraccini, S.; Dalla Via, L.; Gia, O. M.; Passarella, D. Camptothecin-7yl-methanthiole: Semisynthesis and Biological Evaluation. ChemMedChem 2012, 7, 2134-2143.

(24) Ricci, C. G.; Netz, P. A. Docking studies on DNA-ligand interactions: building and application of a protocol to identify the binding mode. J. Chem. Inf. Model. 2009, 49, 1925-1935. 
(25) Christodoulou, M. S.; Calogero, F.; Baumann, M.; GarcíaArgáez, A. N.; Pieraccini, S.; Sironi, M.; Dapiaggi, F.; Bucci, R.; Broggini, G.; Gazzola, S.; Liekens, S.; Silvani, A.; Lahtela-Kakkonen, M.; Martinet, N.; Nonell-Canals, A.; Santamaría-Navarro, E.; Baxendale, J. R.; Dalla Via, L.; Passarella, D. Boehmeriasin A as new lead compound for the inhibition of topoisomerases and SIRT2. Eur. J. Med. Chem. 2015, 92, 766-775.

(26) Christodoulou, M. S.; Sacchetti, A.; Ronchetti, V.; Caufin, S.; Silvani, A.; Lesma, G.; Fontana, G.; Minicone, F.; Riva, B.; Ventura, M.; Lahtela-Kakkonen, M.; Jarho, E.; Zuco, V.; Zunino, F.; Martinet, N.; Dapiaggi, F.; Pieraccini, S.; Sironi, M.; Dalla Via, L.; Gia, O. M.; Passarella, D. Quinazolinecarboline alkaloid evodiamine as scaffold for targeting topoisomerase I and sirtuins. Bioorg. Med. Chem. 2013, 21, 6920-6928.

(27) Svec, F.; Teubner, V.; Tate, D. Location of the Second SteroidBinding Site on the Glucocorticoid Receptor. Endocrinology 1989, 125, 3103-3107.

(28) Svec, F. Differences in the interaction of RU 486 and ketoconazole with the second binding site of the glucocorticoid receptor. Endocrinology 1988, 123, 1902-1906. 